\title{
Combining Transmission Kikuchi Diffraction and Scanning Transmission Electron Microscopy for Irradiated Materials Studies
}

\author{
Chad M. Parish ${ }^{1}$, Kun Wang ${ }^{1}$, Philip D. Edmondson ${ }^{1}$, Kurt A. Terrani ${ }^{1}$, Xunxiang Hu ${ }^{1}$, Rachel L. \\ Seibert $^{2}$ and Yutai Katoh ${ }^{1}$ \\ 1. Oak Ridge National Laboratory, Oak Ridge, TN, USA \\ 2. Illinois Institute of Technology, Chicago, IL, USA
}

Transmission Kikuchi diffraction (tKD) has exploded into the characterization field over the last few years [1], and we have found it to be extraordinarily useful to examine neutron-, ion- and plasmairradiated materials here at ORNL's Low Activation Materials Development and Analysis (LAMDA) laboratory $[2,3]$. Advanced nanostructured materials, intended to provide improved radiation tolerance, and materials that develop nanostructures under irradiation, benefit from high-resolution characterization to understand their mechanical, thermal, or other properties. We examined many irradiated materials using tKD, and have combined tKD with scanning/transmission electron microscopy (S/TEM), primarily X-ray mapping and multivariate statistical analysis (MVSA). We used a JEOL J6500F SEM with EDAX Hikari I EBSD system for non-radioactive materials, and an FEI Versa3D FIB-SEM with Oxford Nordlys EBSD system for both radioactive and non-radioactive materials, and an FEI Talos F200X ChemiSTEM system for S/TEM and X-ray mapping [4,5].

As one example, tungsten (a potential plasma-facing material in fusion) develops massive transmutation $\left(\mathrm{W} \rightarrow \mathrm{Re} \rightarrow \mathrm{Os}\right.$ ) under neutron irradiation. We examined $\sim 700^{\circ} \mathrm{C}, 2 \sim 3$ dpa high-purity polycrystalline tungsten (irradiated in HFIR, a light-water mixed-spectrum reactor). The thermal neutrons from this irradiation bred several atom\% of each Re and Os from the high-purity tungsten, and the displacement damage drove significant irradiation-induced microstructural processes. STEM-EDS shows grain boundary Re-Os precipitates, intragranular Re-Os precipitates, voids (coated in Re-Os), and grainboundary segregation of Re-Os, Figure 1. TEM analysis of the precipitates is complicated by their small size, complex structures, and severe damage to the matrix that causes significant electron scattering (Figure 2). However, tKD can find the identities of the larger precipitates and grain boundary misorientations, allowing us to draw conclusions about the radiation-induced segregation. It is important to note that these samples gave radiation doses (beta-gamma) of $\sim 10-30 \mathrm{mrem} / \mathrm{hr} @ 30 \mathrm{~cm}$, despite being small $\left(\sim 2 \mathrm{~mm}^{3}\right)$. However, the FIB-lift out samples showed no detectable activity. tKD of the same region as Figure 1, shown in Figure 3, allows identification of the large grain-boundary precipitates [likely chi $(\mathrm{Re}, \mathrm{Os})_{3} \mathrm{~W}, \alpha-\mathrm{Mn}$ structural prototype, $\mathrm{I} \overline{4} 3 \mathrm{~m}$ ], and the misorientation axis-angle pairs of the grain boundaries are seen to have no relation to the radiation-induced segregation, which is an important result for interpreting grain-boundary cohesion and mechanical properties.

In this presentation, we will present a number of different examples of combined tKD and STEM methods on irradiated materials, such as tungsten, silicon carbide, advanced steels for GenIV/fusion applications, and high-burnup fuel from a commercial reactor [6].

\section{References:}

[1] G.C. Sneddon et al, Mat Sci Eng R 110 (2016), p. 1.

[2] C. M. Parish et al, Scripta Mat. 127 (2017), p. 132. 
[3] C. M. Parish, K. Wang, and P. D. Edmondson, Scripta Mat. Viewpoint, in press.

[4] C.M. Parish et al, Microsc. Microan. Conf. Proc. 21 (2015), p. 1003.

[5] C.M. Parish, MT3FT-15OR0204122, http://www.osti.gov/servlets/purl/1234344/.

[6] This work is supported by an Early Career Research Program award, US Department of Energy, Office of Science, Fusion Energy Sciences, under contract number DE-AC05-00OR22725. Silicon carbide and fuel work supported by Fuel Cycle R\&D, Office of Nuclear Energy, US DOE. FEI Talos F200X S/TEM provided by the Department of Energy, Office of Nuclear Energy, Fuel Cycle R\&D Program and the Nuclear Science User Facilities.

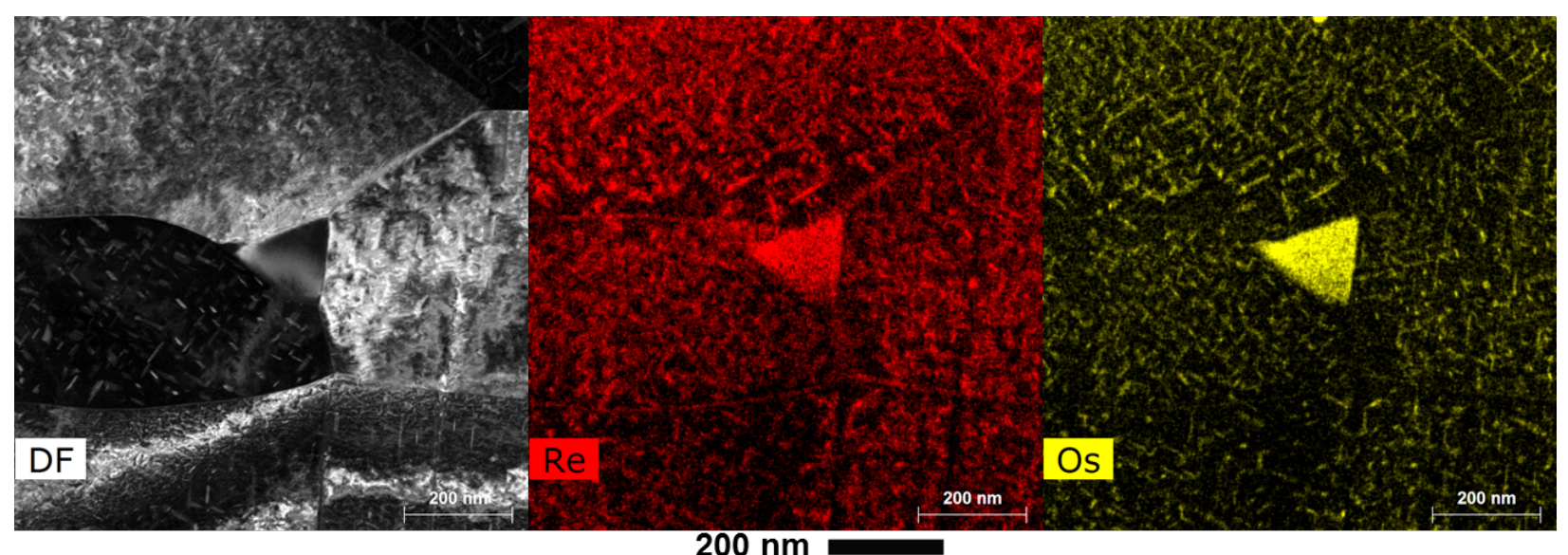

Figure 1. Low-angle annular dark field (DF) and Re and Os X-ray maps of neutron-irradiated tungsten. $\mathrm{Re}+\mathrm{Os} \sim 12 \mathrm{at} \%$, balance W. $\left(\sim 700^{\circ} \mathrm{C}, \sim 2-3 \mathrm{dpa}\right.$.)

Figure 2. Low-angle annular dark field image of radiation-induced precipitates, cavities, and radiation damage in neutronirradiated tungsten. $\left(\sim 700^{\circ} \mathrm{C}, \sim 2-3\right.$ dpa. $)$

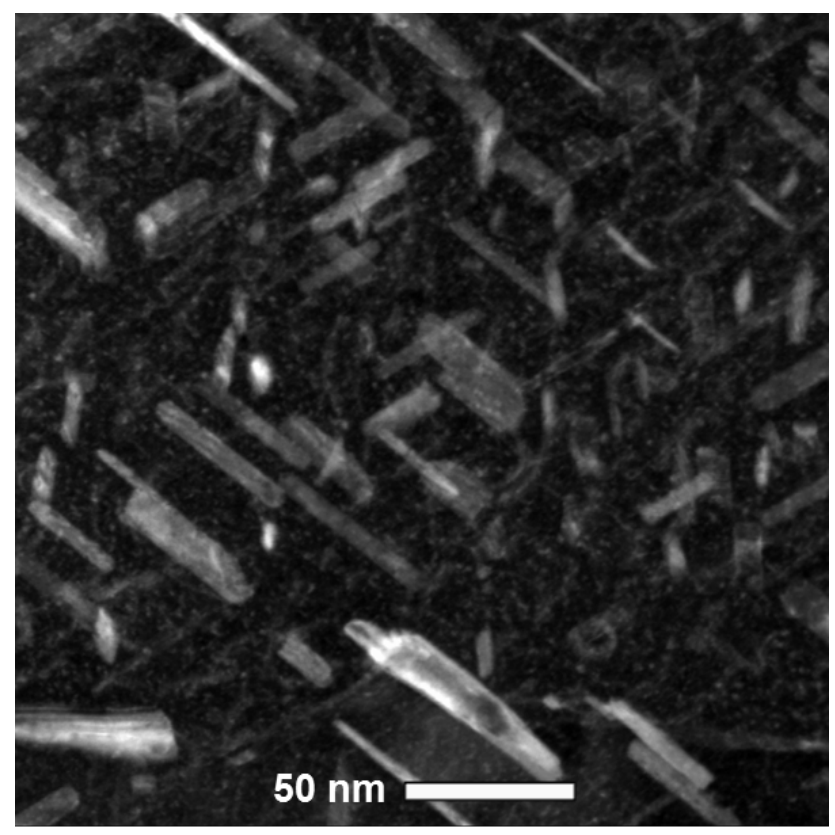

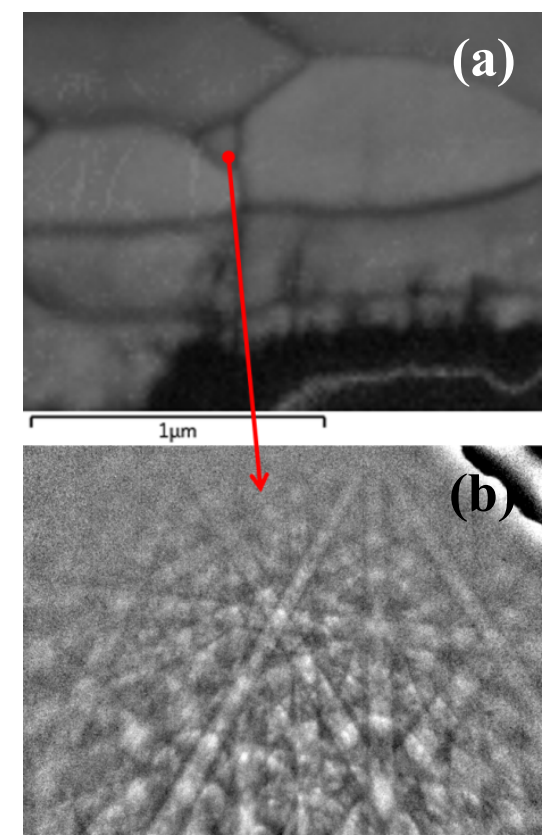

Figure 3. (a) tKD band contrast of Figure 1 region. The precipitate (tentatively $\mathrm{Re}_{3} \mathrm{~W} \chi$ ), has a pattern extracted (b). (neutron-irrad. tungsten, $\sim 700^{\circ} \mathrm{C}, \sim 2-3 \mathrm{dpa}$.) 